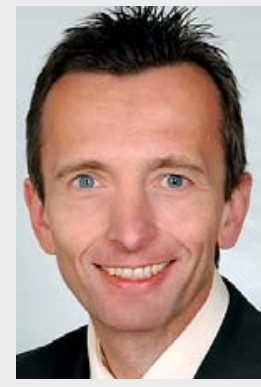

Prof. Dr. med. Michael Schädel-Höpfner

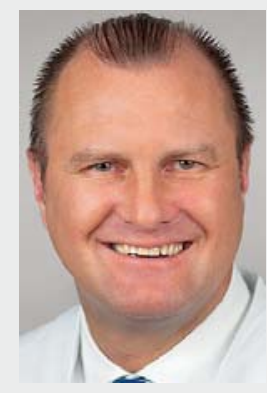

Prof. Dr. med. Dr. med. habil. Michael Sauerbier

\section{Handchirurgie Scan - Aktuelle Publikationen für Sie gescannt}

\author{
Liebe Leserinnen und Leser,
}

in der vor Ihnen liegenden, aktuellen Ausgabe von Handchirurgie Scan finden Sie wie gewohnt eine sorgfältig zusammengestellte Auswahl relevanter und interessanter handchirurgischer Publikationen der letzten Monate. Wir haben diese Artikel aus dem großen Spektrum der zahlreichen Zeitschriften ausgewählt, welche sich ausschließlich oder unter anderem mit der Handchirurgie beschäftigen.

Wie gewohnt stellen wir Ihnen in der Rubrik „Diskussion“ Artikel vor, die sich durch einen besonders innovativen oder kontroversen Inhalt auszeichnen. Erneut haben wir handchirurgische Experten gebeten, diese Publikationen kritisch zu beurteilen und zu kommentieren. Zwei dieser Arbeiten beschäftigen sich mit der operativen Behandlung der Rhizarthrose. Vorgestellt wird zum einen die Technik der arthroskopischen Synovialektomie mit autologer Fettgewebsinterposition, die zumindest in Frühstadien bei kleinen Fallzahlen erfolgversprechende Ergebnisse zeigte. Zum anderen wurde in einer weiteren Publikation über die jetzt mittelfristigen Resultate der Interpositionsarthroplastik mit dem bioresorbierbaren Regjoint-Spacer berichtet, für den gute klinische Ergebnisse auffälligen Röntgenbildern mit deutlichen Osteolysen gegenüberstehen. In Zeiten der winkelstabilen palmaren Plattenosteosynthese von distalen Radiusfrakturen scheint das Komplexe Regionale Schmerzsyndrom seltener aufzutreten - in einer Studie wurde dieser Fragestellung mit einer hohen Fallzahl, aber unbefriedigendem Ergebnis nachgegangen. Dass selbst systematische Literaturanalysen methodisch problematisch sein können und bei der Beuteilung ihrer Schlussfolgerungen Vorsicht geboten ist, zeigt eine Untersuchung zur Bedeutung von Frakturen des Ulnastyloids auf das Outcome von distalen Radiusfrakturen. Ebenfalls mit einer hohen Fallzahl wurde der Häufigkeit von Revisionsoperationen nach Behandlung von Radiuskopffrakturen durch Osteosynthese oder Prothese nachgegangen - mit Ergebnissen, die eine noch höhere Sorgfalt für Diagnostik und Indikationsstellung dieser Verletzungen nahelegen. Ein neuer Ansatz mit diskutablem Effekt ist die Reizosteotomie des distalen Radius bei der minimal-invasiven Verschraubung von Skaphoidpseudarthrosen. Keine Zweifel bestehen aber daran, dass die Sonografie in der Hand des Geübten ein hilfreiches diagnostisches Tool zur Beurteilung von Frühstadien einer Beugesehnenscheiden-Infektion ist.

In der Rubrik „Aktuell“ nehmen erneut Publikationen zu Handgelenk und Handwurzel einen breiten Raum ein. Einen zweiten Schwerpunkt bilden die Nervenkompressionssyndrome. Unter den insgesamt 33 referierten Artikeln finden sich viele weitere interessante Arbeiten, u.a. zu Nervenverletzungen, Infektionen, distalen Radiusfrakturen und Verbrennungen.

Die Rubrik „Weiterbildung“ wird diesmal ganz von der detaillierten Darstellung der freien Fibulatransplantation zur knöchernen Rekonstruktion an der oberen Extremität eingenommen. Dieser umfassende CME-Beitrag wird von uns ausdrücklich empfohlen.

Wir wünschen Ihnen eine spannende und unterhaltsame Lektüre der neuen Ausgabe von Handchirurgie Scan.

Mit herzlichen Grüßen

Ihre Herausgeber

Prof. Dr. med. Michael Schädel-Höpfner

Prof. Dr. med. Dr. med. habil. Michael Sauerbier 